Salinity gradient controls microbial community structure and assembly in coastal solar salterns

Tianran Song ${ }^{1}$, Qiyun Liang ${ }^{2}$, Zhaozhong $\mathrm{Du}^{2}$, Xiaoqun Wang ${ }^{2}$, Guan-Jun Chen², Zong-Jun $\mathrm{Du}^{2}$, Da-Shuai $\mathrm{Mu}^{1,2 *}$

${ }^{1}$ SDU-ANU Joint Science College, Shandong University, Weihai 264209, PR China

${ }^{2}$ College of Marine Science, Shandong University, Weihai 264209, PR China

Running title: Microbial community assembly in the solar saltern

*Address correspondence to:

Da-Shuai Mu, E-mail: dashuai.mu@s,sdu.edu.cn; Tel. (+86) 06315688303. Mailing address: College of Marine Science, Shandong University (Weihai), 180 Wenhuaxi Road, Weihai, Box 264209, PR China

\title{
Conflict of Interest
}

No conflict of interest exists in the submission of this manuscript, and the manuscript has been approved by all authors for publication. 


\section{Abstract}

Salinity acts as a critical environmental filter on microbial communities in natural systems, negatively affecting microbial diversity. However, how salinity affects the community assembly remains unclear. This study used Wendeng multi-pond saltern as a model to evaluate the prokaryotic community composition and diversity and quantify the relative importance of ecological processes across salinity gradients. Results showed that low saline salterns $(45-80 \mathrm{~g} / \mathrm{L})$ exhibited higher bacterial diversity than those in high saline salterns $(175-265 \mathrm{~g} / \mathrm{L})$. The relative abundance of taxa assigned to Halanaerobiaceae, Haloferacaceae, Desulfohalobiaceae, Phormidiaceae, Rohodobacteraceae, and Nitrococcaceae was higher with increasing salinity. Salinity and $\mathrm{pH}$ were the primary environmental factors that directly or indirectly determined the composition and diversity of prokaryotic communities. Microbial co-occurrence network dynamics were more complex in the sediment than in water of salterns. An infer Community Assembly Mechanisms by Phylogenetic-bin-based null model analysis (iCAMP) showed that microbial community assembly in sediment and water differed. Our findings provide more information about microbial community structure and the importance of various ecological processes in controlling microbial community diversity and succession along salinity gradients in water and sediment environments. Keywords: multi-pond saltern; salinity gradients; microbial community; assembly; ecological processes

\section{Introduction}

Salinity acts as a critical environmental filter on microbial communities in 
ecosystems [1,2]. In soil environments, many studies indicated that soil salinization could significantly influence biological structures and functions [3]. Soil salinity limits water availability to plants and microorganisms, thus acting as a stressor. In aquatic environments, salinity was a critical factor in shaping microbial diversity and community structure [4]. At a global scale, salinity has been demonstrated to be one of the essential factors affecting microbial distribution [5].

Some studies showed that salinity is the crucial environmental filtering in the assembly of soil microbial communities along with salinity gradients [3,6]. However, whether or how salinity gradients affect the microbial community assembly remains unknown in aquatic and sediment environments. Understanding community assembly processes is vital to find the potential factors governing microbial community structure [7,8]. Unraveling the drivers controlling community assembly is a central issue in ecology [8]. It is known that both deterministic (e.g., homogeneous selection and heterogeneous selection) and stochastic processes (e.g., homogenizing dispersal, dispersal limitation, and 'drift') affect the assembly of microbial communities. Deterministic processes refer to environment filtering or biotic interactions, while stochastic processes refer to passive dispersal and random demographic changes in mortality [7,8]. By examining deviations from infer Community Assembly Mechanisms by Phylogenetic-bin-based null model analysis (iCAMP), changes in the relative importance of various processes for microbial communities can be quantified [9]. Recent studies investigated community assembly processes along with aridity [10] and $\mathrm{pH}$ [11] gradients, but little is known about microbial community assembly processes 
along a salinity gradient.

Multi-pond salterns are semi-artificial coastal systems designed to harvest $\mathrm{NaCl}$ from seawater. In this system, seawater is pumped through multi-shallow ponds, in which seawater is gradually driven to ponds of greater salinities, ranging from that of seawater to sodium chloride saturation and sometimes even beyond [12]. These systems are well known as continuous or semi-continuous systems because each set of ponds maintains a range of salinity for a relatively long time. Many ecological changes happen through this gradient; for example, the biodiversity decreases with the salinity increasing [12]. There are many ecological and microbiological studies on the ecology of multi-pond salterns, to the extent that these salterns can be used as model systems for the variations induced by the environmental factor [13].

In multi-pond salterns, salinity could play an important role in microbial community composition and found that microbial diversity decreased as salinity increased [14]. However, this pattern is not consistently observed for all microbial communities [15]. It is a long-standing goal and challenge for ecologists to understand better the microbial taxonomic composition and diversity in multi-pond salterns and the mechanisms that shape community structure [16]. In microbial ecology, it has been proposed that "everything is everywhere, but the environment selects," which suggests that a variation in environmental factors could drive the biogeographic patterns of microbial community composition [17]. Microbial diversities or communities are altered by environmental factors such as temperature, salinity, and biological factors [18,19]. In some ecosystems, community composition changes quickly along with the rapid 
changing of environmental factors. These changes may reflect rapid growth or dispersal of rare or dormant taxa from a "seed bank" [20,21]. Many studies indicate that dispersal between distant environments is limited [22,23], implying that microbial communities could also be governed by their demographic history.

The Wendeng solar saltern is a multi-pond saltern that originated from seawater and comprises a set of shallow ponds, where the water gradually evaporates and salts concentrate. Salinities (35 to $300 \mathrm{~g} / \mathrm{L}$ ) and physicochemical factors in these solar salterns vary greatly. They offer the best possible opportunity to rule out the geographical isolation effect, thereby providing a valuable model to investigate the relationship between microbial community composition and various environmental factors in solar salterns.

The objectives of this study were to (a) compare the microbial community composition and diversity in multi-pond solar salterns using 16S rRNA gene amplicons; (b) evaluate the distribution patterns of the microbial community composition and across entire prokaryotic communities along with salinity and other environmental factors; and (c) determined how salinity affects microbial community assembly processes in water and sediments.

\section{METHODS AND MATERIALS}

\section{Sample collection}

In May 2019, samples were obtained from Wendeng multi-pond saltern (Weihai, China) and included samples from five ponds with salinity of $45,80,125,175$ and 265\%

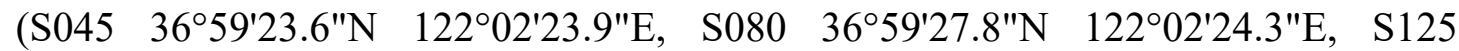




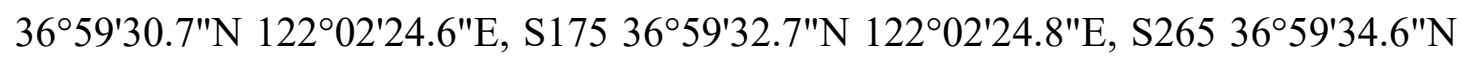
$122^{\circ} 02^{\prime} 24.9^{\prime \prime E}$ ). Sediment and water samples were taken from each pond (Fig. 1). For every pond, water salinity and $\mathrm{pH}$ values were measured in situ [12]. Each pond was sampled in three random locations by collecting sediment and water samples. The water samples from the same pond were pooled and concentrated from 3 liters to $500 \mathrm{~mL}$ by a hollow fiber membrane module (pore size: $0.22 \mu \mathrm{m}$ ), and then collected in a $500 \mathrm{~mL}$ sterile opaque polypropylene bottle; the sediment samples from the same pond were pooled by $500 \mathrm{~mL}$ sterile water. After collection, all samples were sent to the laboratory instantly and kept at $4{ }^{\circ} \mathrm{C}$ during the transportation and stored at $-80{ }^{\circ} \mathrm{C}$ after treatment.

\section{The measurement of physicochemical factors}

Place the sediment sample in a Petri dish and dry it at $105^{\circ} \mathrm{C}$ for $6 \mathrm{~h}$. After air drying, the sediment extract was obtained in the ratio of water to soil of $2.5: 1$, and the $\mathrm{pH}$ of the sediment was measured by measuring the extract. Weigh $5 \mathrm{~g}$ of dried sediment sample, put it into a $50 \mathrm{ml}$ centrifuge tube, add $25 \mathrm{ml}$ of water and shake for $3 \mathrm{~min}$ to obtain a 5:1 water-soil extraction. Then the water-soil extraction and water samples from salterns were filtered by $0.22 \mu \mathrm{m}$ polyether sulfone membranes. The soluble ion (including $\mathrm{Cl}^{-}, \mathrm{Br}^{-}, \mathrm{SO}_{4}^{2-}, \mathrm{Na}^{+}, \mathrm{NH}^{4+}, \mathrm{K}^{+}, \mathrm{Mg}^{2+}$ and $\mathrm{Ca}^{2+}$ ) concentration was measured by ICS-1100 (Thermo, USA).

\section{Genomic DNA extraction and sequencing}

All treated samples, as described above, were centrifuged to remove particulate matter, and the supernatant fraction was filtered by polyether sulfone membranes to obtain microorganisms with a size greater than $0.22 \mu \mathrm{m}$. These membranes were kept at 
$-80{ }^{\circ} \mathrm{C}$ before use. Equivalent volumes of samples (with different salinities) were dissolved prior to DNA extraction using a FastDNA Spin Kit for soil (MP Biomedical, France). The prime set composed of 338F (5'-ACTCCTACGGGAGGCAGCAG-3') and 806R (5'-GGACTACHVGGGTWTCTAAT-3') were selected for the microbial community structure analysis. Sequencing was carried out on a MiSeq PE300 platform at the Shanghai Majorbio Bio-pharm Technology Co.,Ltd (Shanghai, China).

\section{Sequence analysis}

The sequences were demultiplexing, quality trimming, denoising and removing chimeric by an automatic pipeline, Amplicon Sequence Analysis Pipeline (ASAP2) [24]. Operational taxonomic units (OTUs) were clustered on basis of cut value $97 \%$ and taxonomic annotation was obtained on basis of SILVA_138_SSU_RefNR99 database [25,26], which were analyzed by vsearch v1.2.11 [27]. A phylogenetic tree of OTUs was constructed by using FastTree [28].

The alpha-diversity (Shannon-Wiener and Simpson diversity indexes) and Venn diagrams (showing shared and unique OTUs) were calculated by R package "microeco" [29]. TukeyHSD test of ANOVA between alpha diversities of sediment and water samples were employed. Meanwhile, for distinguishing the general distribution patterns of the prokaryotic community composition among sediment and water samples of salterns, the Non-metric multidimensional scaling (NMDS) was performed on basis of Bray-Curtis distance by R package "vegan" [30]. Further, to evaluate the linkages between the prokaryotic community structure and environmental parameters, the Mantel test and redundancy analysis (RDA) were performed by R package "microeco" 
[29]. LEfSe was employed for illustrating different taxa between sediment and water groups of salterns [31] and calculated by R package "microeco" [29].

Microbial ecological networks (MENs) were constructed by using the Molecular Ecological Network Analysis Pipeline (MENAP) (http://ieg4.ou.edu/MENA/) to reveal the possible co-occurrence patterns [32,33]. Random matrix theory (RMT) threshold was set as 0.85 to construct MENs. For each node, its within-module connectivity $\left(Z_{i}\right)$ and among-module connectivity $\left(P_{i}\right)$ [34] were calculated and used for the classification of its topological roles in the network. To identify the keystone taxa, the following simplified classification was established: (i) peripheral nodes $\left(Z_{i} \leq 2.5, P_{i} \leq 0.62\right)$, which possessed only a few links that were almost always associated with nodes within their modules; (ii) connectors $\left(Z_{i} \leqslant 2.5, P_{i}>0.62\right)$, which were highly connected to several modules; (iii) module hubs $\left(Z_{i}>2.5, P_{i} \leq 0.62\right)$, which were highly connected to numerous microbes in their own modules; and (iv) network hubs $\left(Z_{i}>2.5, P_{i}>0.62\right)$, which acted as both module hubs and connectors. Module hubs, connectors and network hubs are referred to as keystone nodes [35,36]. 1000 corresponding random networks were generated with the same network size and an average number of links for each network. The visualization of MENs was performed by R package "ggraph" (https://github.com/thomasp85/ggraph).

To investigate the assembly mechanisms of different microorganism groups, the Infer Community Assembly Mechanisms by Phylogenetic-bin-based null model (iCAMP, https://github.com/DaliangNing/iCAMP1) was employed [37]. By using iCAMP, five assembly mechanisms of different microorganism groups, including homogeneous 
selection (HoS), heterogeneous selection (HeS), dispersal limitation (DL), homogenizing dispersal (HD), and drift (DR). Besides, the variation between sediment and water groups of salterns in HoS and DL were investigated in this study. The statistical difference test of stochasticity estimated between sediment and water groups was calculated by using the Mann-Whitney U test.

\section{Nucleotide sequence accession numbers}

During this study, the 16S rRNA gene data sets of Wendeng salterns have been deposited in the Sequence Read Archive under accession numbers PRJNA559148 and PRJNA799174 for all the samples.

\section{Results}

\section{General features for $16 \mathrm{~S}$ rRNA gene sequences and the taxonomic compositions of the prokaryotic communities.}

After sequences filtering, clean data were obtained for a total of 575,279 sequences (length distribution of valid sequences were 327-402 bp), generating 5,379 OTUs. The 14 most abundant phyla, most of which comprised $83.73 \sim 90.89 \%$ and $90.65 \sim 98.52 \%$ of the prokaryotic community composition in sediment and water samples, which were obtained from Wendeng salterns (Fig. 1), respectively (Table S1). The phyla Bacteroidota and Proteobacteria were most abundant in sediment and water samples in salterns; by contrast, the phyla Chloroflexi and Halobacterota were more abundant in sediment than that in water samples. Down to the order level, the relative abundance of the top 35 abundant order taxa in water samples of salterns comprised huger prokaryotic community composition than that in sediment samples (Fig. 2). In detail, 
the relative abundance of order Halobacterales, Halanaerobiales and Desulfovibrionales were respectively appropriately $23 \%, 17 \%$ and $12 \%$ in sediment sample (salinity $265 \mathrm{~g} / \mathrm{L}$ ), performing a high salinity dependence; by contrast, the relative abundance of order Chloroplast in water samples (salinity 45 and $80 \mathrm{~g} / \mathrm{L}$ ) remained at about $7.3 \%$ (range $7.1 \sim 7.5 \%$ ) and diminished to $<1 \%$ abundance along with increasing salinity, indicating a negative dependence with salinity. The variation of order Rhodobacterales along with increasing salinity in water samples exhibited similar patter with order Chloroplast. The relative abundance variation of order Izemoplasmatales performed a hump-shaped pattern in water samples at salinity 80 $\sim 175 \mathrm{~g} / \mathrm{L}$. The variation of order Burkholderiales exhibited a sharply huger humpshaped pattern in water sample at salinity $80 \mathrm{~g} / \mathrm{L}$. Meanwhile, the relative abundance variation of order Balneolales and Cyanobacteriales also shaped hump patterns in sediment samples at salinity 125 and $175 \mathrm{~g} / \mathrm{L}$.

\section{Diversity of prokaryotic communities and the relationships with physicochemical}

\section{factors}

The alpha-diversities (Shannon and Simpson indexes) differed significantly between sediment and water samples, obtained from Wendeng salterns along a gradient increasing salinity (Fig. 3A). Meanwhile, the alpha-diversities in the same salinity ponds also exhibited a significant difference between sediment and water samples (Table 1). On basis of NMDS, the successions of prokaryotic communities along increasing salinity performed a very similar pattern between sediment and water samples (Fig. 3B). The community structures were also easily distinguished between 
sediment and water samples. The shared OTUs in sediment samples were more than water samples (Fig. S1A-B). By contrast, the shared OTUs at the same salinity ponds exhibited a diminishing tendency along with increasing salinity (Fig. S1C-G). The significantly differential abundant family taxa differed at the same salinity ponds (Fig. S2 and S3). The family Woeseiaceae, Cyclobacteriaceae, Pirellulaceae, Desulfocapsaceae and Trueperaceae were differential abundant taxa in sediment sample at salinity $45 \mathrm{~g} / \mathrm{L}$; by contrast, the family Rhodobacteraceae, Cryomorphaceae and Clade_I_o_SAR11 were differential abundant taxa in sediment sample at salinity $45 \mathrm{~g} / \mathrm{L}$. Meanwhile, the family Rhodobacteraceae was also differential abundant taxa in the sediment sample at salinity $175 \mathrm{~g} / \mathrm{L}$. The family Haloferacaceae was differential abundant taxa in sediment and water samples at salinity $265 \mathrm{~g} / \mathrm{L}$.

To further illustrate the variation of prokaryotic communities, we measured a series of physicochemical factors (Table S2). On basis of RDA, salinity and pH were more important factors in sediment samples, which influenced the community structure at salinity $265 \mathrm{~g} / \mathrm{L}$ (Fig. 4A-B and Table S3). The community structure of sediment and water samples at the same salinity ponds were influenced by different physicochemical factors. The genus Natronomonas, Thiohalorhabdus, Halapricum and Salinibacter performed a significantly positive relationship with salinity and $\mathrm{pH}$ in sediment samples; by contrast, the genus Woeseia, g_Yoonia-Loktanella and Desulfotignum showed a significantly negative relationship with salinity (Fig. 4C). Interestingly, the genus Halopeptonella, Salinibacter, Halorubrum, Halobellus and Halonotius exhibited a significantly positive relationship with salinity, but a negative association with $\mathrm{pH}$ in 
water samples; by contrast, the genus Roseovarius and Litoricola performed a significantly negative relationship with salinity, but positive relationship with $\mathrm{pH}$ (Fig. 4D). Most alpha-diversity had a significantly negative relationship with salinity and $\mathrm{pH}$ in sediment samples (Fig. S4A), but a significantly positive relationship with $\mathrm{pH}$ in water samples (Fig. S4B). Meanwhile, most alpha-diversity had a significantly negative relationship with physicochemical factors, except $\mathrm{pH}$ and $\mathrm{Ca}^{2+}$ (Fig. S4B).

\section{The characters of MENs}

We constructed MENs of sediment and water samples (Fig. 5A-B) on basis of Pearson correlations of log-transformed operational taxonomic unit (OTU) abundances. The empirical MENs of both sediment and water samples were significantly different from random MENs and all empirical MENs exhibited scale-free features (Table S4). Degree distributions of MENs both in sediment and water samples followed the powerlaw distribution, indicating "rich get richer". MENs of sediment samples were more complex than that of water samples. MENs of water samples possessed higher average degree (avgK), average clustering coefficient (avgCC), average path distance (APD), graph density (GD), transitivity (Trans) and positive links along with diminishing networks composition.

The putative roles of network nodes were confirmed on basis of their within-module connectivity $\left(Z_{i}\right)$ and participation coefficient $\left(P_{i}\right)$ (Table S4). Most nodes were identified as peripheral $(91.3 \%, 619 / 678)$, and the remaining nodes were module hubs and connectors. Due to the contribution of module hubs and connectors to network topology, module hubs and connectors have been proposed to represent potential 
keystone taxa. The keystone taxa of MENs in sediment samples was more than that of MENs in water samples (Fig. 5C-F). The bulk of keystone taxa of MENs in sediment samples was affiliated to family Rhodobacteraceae and Flavobacteriaceae (27.5\%, 11/40). The family, to which keystone taxa in MENs of sediment were affiliated, was more diverse than water samples (Table S4; 25:6). The family Woeseiaceae, reported as the most abundant taxa in marine sediment, could contribute to network topology as module hubs of MENs in sediment samples.

\section{Dispersal limitation and homogeneous selection shape prokaryotic communities in}

\section{saltern sediment}

We employed an iCAMP to infer community assembly mechanisms and found that dispersal limitation (DL) and homogenous selection (HoS) were the key processes driving prokaryotic community assembly in saltern sediment, but HoS was the most essential process in saltern water (Fig. 6A-B). DL had a more significant effect on community assembly in saltern sediment (43.2\%), followed by $\operatorname{HoS}(29.2 \%)$; by contrast, HoS was the most prominent effect in saltern water (61.1\%). The variation of HoS in saltern sediment exhibited a rising trend along with increasing salinity, but a slightly diminishing tendency in saltern water (Fig. 6C). The changes of DL performed a slightly rising trend along with salinity but maintained at low contribution to community assembly (Fig. 6D). Estimated stochasticity between saltern sediment and water samples exhibited significant differences on basis of the Mann-Whitney U test (Fig. 6E).

\section{DISCUSSION}


The effects of salinity on the structure of the prokaryotic community are mainly confined to solar saltern ponds, salt lakes, dynamic estuaries, and vertical water columns. Some studies have focused on multi-pond solar salterns with different salinity levels $[12,38,39]$. However, these reports did not focus on how salinity affects microbial community assembly processes. In this study, we determined the effects of environmental factors on the dynamic of the prokaryotic community composition in sediment and water samples from multi-pond solar salterns. Our results showed that the understanding of the precise microbial structure patterns in response to gradients of salinity and the microbial community assembly rules would have been improved in this study by conducting a deep analysis involving different sources of sample (water and sediments), environmental parameters, and quantifying the relative importance of ecological processes.

The diversity of prokaryotic communities in a low-salinity saltern was higher than that in a high-salinity saltern, consistent with the common ecological principle which extreme environments have a low community diversity $[12,40]$. The possible explanation for this negative effect could be attributed to the fact that the accumulation of salt in water and sediment environments elevates the extracellular osmolarity [41], and microorganisms that fail to adapt to osmotic stress may die, thus reducing microbial alpha diversity. Variation in microbial community structure of solar saltern was also mainly explained by salinity in this study, which is consistent with the results found in estuarine and marine environments [15]. In contrast, this diversity did not decrease as increasing salinity for low salinity values of $<80 \mathrm{~g} / \mathrm{L}$ (Table S1). Like other studies, the 
prokaryotic community diversities did not decrease with the salinity increasing with a range of salinities (0-100 g/L) in salt lakes [4,42]. Furthermore, the prokaryotic community diversities in sediment samples were much higher than those in water samples, and similar results were reported in Tunisian multi-pond solar saltern [38]. This may have occurred because the sediment contained a stable and nutrient-rich environment.

The related diverse between communities in water and sediments were also shown in network analysis. Microbial network analysis can improve our perspectives on ecological processes and complex interaction webs beyond microbial community composition and richness [43]. Microbial co-occurrence network dynamics are more complex in sediment than in the water of salterns. One potential reason was that communities in sediments had a higher Simpson and Shannon index than water samples. A previous study which focused on the co-occurrence networks in a mountain ecosystem also found that low bacterial diversity had low network complexity, which supported our results [44]. Another potential reason was that sediment contained a stable environment, while water in salterns might be frequently affected by tides and sun exposure. Indeed, studies showed that eukaryotic plankton co-occurrence networks were influenced by distinct environmental factors in reservoirs[45]. In the network, keystone taxa have been frequently referred to as "ecosystem engineers" owing to their enormous influence in the community[46]. In the saltern system, sediment harbored much more keystone taxa, most of which belonged to Woeseiaceae, Rhodobacteraceae, and Flavobacteriaceae. Among these groups, Woeseiaceae has been identified as an 
abundant core member of microbial communities in global marine sediments $[47,48]$, suggesting that Woeseiaceae might have a large range of adaption to salinity.

The community assembly mechanism is one of the most compelling questions in ecology, and previous studies have indicated that assembly mechanisms mainly include deterministic and stochastic processes [8]. The deterministic processes included homogeneous selection (HoS) and heterogeneous selection (HeS), while the stochastic processes were divided into dispersal limitation (DL), homogenizing dispersal (HD), and drift (DR) [8]. It was commonly known that salinity imposed an intense selection pressure on the microbial community, which resulted in a dominance of deterministic processes in the coastal wetland[1] and desert[3] ecosystems. However, our study found that different from the saltern water system and other soil ecosystems studies[1,3], salinity could impose strong dispersal limitation processes on the microbial community of saltern sediment samples. One possible reason was that microorganisms in the sediment of different saltern pools had a poor dispersal ability than water. Another reason is that sediment has a stable and nutrient-rich environment, salt-tolerant microorganisms' growth could respond rapidly to environmental changes, thereby reducing the environmental heterogeneity gradient.

Microbial biodiversity studies have led to two major conflicting hypotheses [49]. One is the "seed bank" hypothesis, which suggests that microorganisms are ubiquitous and have few barriers to gene flow, resulting in similar microbial communities across different spatial scales and habitats $[21,50]$. The other one is the "barriers to dispersal" hypothesis, which shows similar patterns in animals or plants, suggesting that 
microorganism's differentiation is governed by geographic barriers or ecological barriers $[22,51]$. Many studies reported the relationship between microbial community diversity and different hypersaline environments [4,52], supporting the "barriers to dispersal" hypothesis. As one of the research of microbial community diversity in different hypersaline backgrounds, our study reported for the presence of members of Halobacterota, Proteobacteria, Bacteroidota, Planctomycetota, Spirochaetes, Nanohaloarchaeota, members of AC1, Atribacteria, BRC1, Chloroflexi, DeinococcusThermus group, Gracilibacteria, Hydrogenedentes, Ignavibacteriae, KSB3 (Modulibacteria), Latescibacteria, SAR406 clade, and Thermotogae in sediment and water samples of solar salterns. Some common members of prokaryotic communities were also reported in other hypersaline environments, such as Deinococcus-Thermus group, BRC1, Spirochaetes, Thermotogae [53,54]. These results showed that different hypersaline environments (e.g., solar salterns and salt lakes) might have some common members of Bacteria or Archaea, which supports the "seed bank" hypothesis. Furthermore, in this study, all of the salterns originated from seawater and comprised a set of shallow ponds, where the water gradient evaporates and salts concentrate. Thus, the initial composition of prokaryotic communities should have been similar in each saltern. However, as the water gradient evaporated and different salinities accumulated, different prokaryotic community compositions appeared in salterns of different salinities (Fig. 3). These results also support the "seed bank" hypothesis, in which "everything is everywhere, but the environment selects"[50]. However, the mechanism driving the "seed bank" requires further study. Additionally, the main composition of 
prokaryotic communities of Wendeng solar salterns differs from those found in other salterns, such as a Greek solar saltern [55]. Therefore, geographic and ecological barriers may be the governing powers that create and maintain biodiversity in the Wendeng solar salterns and are thus reasonable for developing the unique microbial community structure in this unique eco-system.

In aquatic systems, salinity is a major environmental driving force to control prokaryotic communities, such as salt lakes [4], solar saltern ponds [56], and the Baltic Sea [15]. Global studies have found that salinity, rather than other physical and chemical factors, determines microbial community composition [57]. Thus, microbial studies along salinity gradients may provide more clues to the global distribution pattern of microbial communities, depending on salinity changes. In this study, the Mantel test showed that both salinity and $\mathrm{pH}$ were the most critical environmental factors to regulate prokaryotic structure changes in the sediment and water of salterns. Consistent with our reports, many other reports have shown that $\mathrm{pH}$ was the main driving force of microbial community distribution in different ecosystems [58]. The $\mathrm{pH}$ factor has been suggested to be the main factor that integrates the physiochemical status of aquatic ecosystems [59].

In summary, the results showed that the composition of prokaryotic communities and assembly processes were directly or indirectly determined by salinity. Our findings shed light on the distribution pattern of prokaryotic communities and the salinity gradient of the whole community, in a single evolutionary process. This baseline information will help to predict the ecological responses of future environmental changes and help to 
reveal the global distribution of microbial composition and diversity of salinity gradients.

\section{Acknowledgments}

This work was supported by the National Natural Science Foundation of China (41876166), Science and Technology Basic Resources Investigation Program of China (2017FY100302).

\section{References}

1. Zhang, G.L.; Bai, J.H.; Tebbe, C.C.; Zhao, Q.Q.; Jia, J.; Wang, W.; Wang, X.; $\mathrm{Yu}, \mathrm{L}$. Salinity controls soil microbial community structure and function in coastal estuarine wetlands. Environ Microbiol 2021, 23, 1020-1037, DOI:10.1111/1462-2920.15281.

2. Banda, J.F.; Zhang, Q.; Ma, L.; Pei, L.; Du, Z.; Hao, C.; Dong, H. Both pH and salinity shape the microbial communities of the lakes in Badain Jaran Desert, NW China. Science of The Total Environment 2021, 791, 148108, DOI:10.1016/j.scitotenv.2021.148108.

3. Zhang, K.; Shi, Y.; Cui, X.; Yue, P.; Li, K.; Liu, X.; Tripathi, B.M.; Chu, H. Salinity Is a Key Determinant for Soil Microbial Communities in a Desert Ecosystem. mSystems 2019, 4, DOI:10.1128/mSystems.00225-18.

4. Zhong, Z.P.; Liu, Y.; Miao, L.L.; Wang, F.; Chu, L.M.; Wang, J.L.; Liu, Z.P. Prokaryotic Community Structure Driven by Salinity and Ionic Concentrations in Plateau Lakes of the Tibetan Plateau. Appl Environ Microbiol 2016, 82, 18461858, DOI:10.1128/AEM.03332-15.

5. Lozupone, C.A.; Knight, R. Global patterns in bacterial diversity. P Natl Acad Sci USA 2007, 104, 11436-11440, DOI:10.1073/pnas.0611525104.

6. Caruso, T.; Chan, Y.K.; Lacap, D.C.; Lau, M.C.Y.; Mckay, C.P.; Pointing, S.B. Stochastic and deterministic processes interact in the assembly of desert microbial communities on a global scale. Isme $J$ 2011, 5, 1406-1413, DOI:10.1038/ismej.2011.21.

7. Nemergut, D.R.; Schmidt, S.K.; Fukami, T.; O'Neill, S.P.; Bilinski, T.M.; Stanish, L.F.; Knelman, J.E.; Darcy, J.L.; Lynch, R.C.; Wickey, P.; et al. Patterns and Processes of Microbial Community Assembly. Microbiology and Molecular Biology Reviews 2013, 77, 342-356, DOI:10.1128/mmbr.00051-12.

8. Zhou, J.; Ning, D. Stochastic Community Assembly: Does It Matter in Microbial Ecology? Microbiology and Molecular Biology Reviews 2017, 81, DOI:10.1128/mmbr.00002-17. 
9. Ning, D.; Yuan, M.; Wu, L.; Zhang, Y.; Guo, X.; Zhou, X.; Yang, Y.; Arkin, A.P.; Firestone, M.K.; Zhou, J. A quantitative framework reveals ecological drivers of grassland microbial community assembly in response to warming. Nat Commun 2020, 11, 4717, DOI:10.1038/s41467-020-18560-z.

10. Stomeo, F.; Valverde, A.; Pointing, S.B.; Mckay, C.P.; Warren-Rhodes, K.A.; Tuffin, M.I.; Seely, M.; Cowan, D.A. Hypolithic and soil microbial community assembly along an aridity gradient in the Namib Desert. Extremophiles 2013, 17, 329-337, DOI:10.1007/s00792-013-0519-7.

11. Tripathi, B.M.; Stegen, J.C.; Kim, M.; Dong, K.; Adams, J.M.; Lee, Y.K. Soil $\mathrm{pH}$ mediates the balance between stochastic and deterministic assembly of bacteria. Isme J 2018, 12, 1072-1083, DOI:10.1038/s41396-018-0082-4.

12. Benlloch, S.; López-López, A.; Casamayor, E.O.; Øvreås, L.; Goddard, V.; Daae, F.L.; Smerdon, G.; Massana, R.; Joint, I.; Thingstad, F.; et al. Prokaryotic genetic diversity throughout the salinity gradient of a coastal solar saltern. Environmental Microbiology 2002, 4, 349-360, DOI:10.1046/j.14622920.2002.00306.x.

13. Joint, I.; Henriksen, P.; Garde, K.; Riemann, B. Primary production, nutrient assimilation and microzooplankton grazing along a hypersaline gradient. FEMS Microbiol Ecol 2002, 39, 245-257, DOI:10.1111/j.1574-6941.2002.tb00927.x.

14. Oren, A. Molecular ecology of extremely halophilic Archaea and Bacteria. FEMS Microbiol Ecol 2002, 39, 1-7, DOI:10.1111/j.1574-6941.2002.tb00900.x.

15. Herlemann, D.P.; Labrenz, M.; Jurgens, K.; Bertilsson, S.; Waniek, J.J.; Andersson, A.F. Transitions in bacterial communities along the $2000 \mathrm{~km}$ salinity gradient of the Baltic Sea. ISME $J$ 2011, 5, 1571-1579, DOI:10.1038/ismej.2011.41.

16. Webb, C.O.; Ackerly, D.D.; McPeek, M.A.; Donoghue, M.J. Phylogenies and community ecology. Annu Rev Ecol Syst 2002, 33, 475-505, DOI:10.1146/annurev.ecolysis.33.010802.150448.

17. Gibbons, S.M.; Caporaso, J.G.; Pirrung, M.; Field, D.; Knight, R.; Gilbert, J.A. Evidence for a persistent microbial seed bank throughout the global ocean. Proc Natl Acad Sci U S A 2013, 110, 4651-4655, DOI:10.1073/pnas.1217767110.

18. Gilbert, J.A.; Steele, J.A.; Caporaso, J.G.; Steinbruck, L.; Reeder, J.; Temperton, B.; Huse, S.; McHardy, A.C.; Knight, R.; Joint, I.; et al. Defining seasonal marine microbial community dynamics. ISME J 2012, 6, 298-308, DOI:10.1038/ismej.2011.107.

19. Gilbert, J.A.; Field, D.; Swift, P.; Newbold, L.; Oliver, A.; Smyth, T.; Somerfield, P.J.; Huse, S.; Joint, I. The seasonal structure of microbial communities in the Western English Channel. Environ Microbiol 2009, 11, 3132-3139, DOI:10.1111/j.1462-2920.2009.02017.x.

20. Caporaso, J.G.; Paszkiewicz, K.; Field, D.; Knight, R.; Gilbert, J.A. The Western English Channel contains a persistent microbial seed bank. ISME J 2012, 6, 1089-1093, DOI:10.1038/ismej.2011.162.

21. Lennon, J.T.; Jones, S.E. Microbial seed banks: the ecological and evolutionary implications of dormancy. Nat Rev Microbiol 2011, 9, 119-130, 
DOI:10.1038/nrmicro2504.

22. Whitaker, R.J.; Grogan, D.W.; Taylor, J.W. Geographic barriers isolate endemic populations of hyperthermophilic archaea. Science 2003, 301, 976-978, DOI:10.1126/science.1086909.

23. Follows, M.J.; Dutkiewicz, S.; Grant, S.; Chisholm, S.W. Emergent biogeography of microbial communities in a model ocean. Science 2007, 315, 1843-1846, DOI:10.1126/science. 1138544.

24. Tian, R.; Imanian, B. ASAP 2: a pipeline and web server to analyze marker gene amplicon sequencing data automatically and consistently. BMC bioinformatics 2022, 23, 27, DOI:10.1186/s12859-021-04555-0.

25. Quast, C.; Pruesse, E.; Yilmaz, P.; Gerken, J.; Schweer, T.; Yarza, P.; Peplies, J.; Glöckner, F.O. The SILVA ribosomal RNA gene database project: improved data processing and web-based tools. Nucleic acids research 2013, 41, D590D596, DOI:10.1093/nar/gks1219.

26. Yilmaz, P.; Parfrey, L.W.; Yarza, P.; Gerken, J.; Pruesse, E.; Quast, C.; Schweer, T.; Peplies, J.; Ludwig, W.; Glöckner, F.O. The SILVA and "All-species Living Tree Project (LTP)" taxonomic frameworks. Nucleic acids research 2014, 42, D643-D648, DOI:10.1093/nar/gkt1209.

27. Rognes, T.; Flouri, T.; Nichols, B.; Quince, C.; Mahé, F. VSEARCH: a versatile open source tool for metagenomics. PeerJ 2016, 4, e2584, DOI:10.7717/peerj.2584.

28. Price, M.N.; Dehal, P.S.; Arkin, A.P. FastTree: computing large minimum evolution trees with profiles instead of a distance matrix. Molecular biology and evolution 2009, 26, 1641-1650, DOI:10.1093/molbev/msp077.

29. Liu, C.; Cui, Y.; Li, X.; Yao, M. microeco: an R package for data mining in microbial community ecology. FEMS microbiology ecology 2021, 97, DOI:10.1093/femsec/fiaa255.

30. Dixon, P. VEGAN, a package of R functions for community ecology. Journal of Vegetation Science 2003, 14, 927-930, DOI:10.1111/j.16541103.2003.tb02228.x.

31. Segata, N.; Izard, J.; Waldron, L.; Gevers, D.; Miropolsky, L.; Garrett, W.S.; Huttenhower, C. Metagenomic biomarker discovery and explanation. Genome biology 2011, 12, R60, DOI:10.1186/gb-2011-12-6-r60.

32. Deng, Y.; Jiang, Y.-H.; Yang, Y.; He, Z.; Luo, F.; Zhou, J. Molecular ecological network analyses. BMC bioinformatics 2012, 13, 113, DOI:10.1186/1471-210513-113.

33. Zhou, J.; Deng, Y.; Luo, F.; He, Z.; Tu, Q.; Zhi, X. Functional molecular ecological networks. mBio 2010, 1, DOI:10.1128/mBio.00169-10.

34. Guimerà, R.; Nunes Amaral, L.A. Functional cartography of complex metabolic networks. Nature 2005, 433, 895-900, DOI:10.1038/nature03288.

35. Banerjee, S.; Schlaeppi, K.; van der Heijden, M.G.A. Reply to 'Can we predict microbial keystones?'. Nature reviews. Microbiology 2019, 17, 194, DOI:10.1038/s41579-018-0133-x.

36. Röttjers, L.; Faust, K. Can we predict keystones? Nature reviews. Microbiology 
2019, 17, 193, DOI:10.1038/s41579-018-0132-y.

37. Ning, D.; Yuan, M.; Wu, L.; Zhang, Y.; Guo, X.; Zhou, X.; Yang, Y.; Arkin, A.P.; Firestone, M.K.; Zhou, J. A quantitative framework reveals ecological drivers of grassland microbial community assembly in response to warming. Nature communications 2020, 11, 4717, DOI:10.1038/s41467-020-18560-z.

38. Baati, H.; Guermazi, S.; Gharsallah, N.; Sghir, A.; Ammar, E. Novel prokaryotic diversity in sediments of Tunisian multipond solar saltern. Res Microbiol 2010, 161, 573-582, DOI:10.1016/j.resmic.2010.05.009.

39. Dillon, J.G.; Carlin, M.; Gutierrez, A.; Nguyen, V.; McLain, N. Patterns of microbial diversity along a salinity gradient in the Guerrero Negro solar saltern, Baja CA Sur, Mexico. Frontiers in microbiology 2013, 4, 399, DOI:10.3389/fmicb.2013.00399.

40. Fawley, M.W.; Fawley, K.P.; Buchheim, M.A. Molecular diversity among communities of freshwater microchlorophytes. Microb Ecol 2004, 48, 489-499, DOI:10.1007/s00248-004-0214-4.

41. Oren, A. Thermodynamic limits to microbial life at high salt concentrations. Environ Microbiol 2011, 13, 1908-1923, DOI:10.1111/j.14622920.2010.02365.x.

42. Wu, Q.L.; Zwart, G.; Schauer, M.; Kamst-van Agterveld, M.P.; Hahn, M.W. Bacterioplankton community composition along a salinity gradient of sixteen high-mountain lakes located on the Tibetan Plateau, China. Appl Environ Microbiol 2006, 72, 5478-5485, DOI:10.1128/AEM.00767-06.

43. Chen, W.; Wen, D. Archaeal and bacterial communities assembly and cooccurrence networks in subtropical mangrove sediments under Spartina alterniflora invasion. Environ Microbiome 2021, 16, 10, DOI:10.1186/s40793021-00377-y.

44. Li, J.; Li, C.; Kou, Y.; Yao, M.; He, Z.; Li, X. Distinct mechanisms shape soil bacterial and fungal co-occurrence networks in a mountain ecosystem. Fems Microbiol Ecol 2020, 96, DOI:10.1093/femsec/fiaa030.

45. Liu, L.; Chen, H.; Liu, M.; Yang, J.R.; Xiao, P.; Wilkinson, D.M.; Yang, J. Response of the eukaryotic plankton community to the cyanobacterial biomass cycle over 6 years in two subtropical reservoirs. Isme J 2019, 13, 2196-2208, DOI:10.1038/s41396-019-0417-9.

46. Deng, Y.; Jiang, Y.H.; Yang, Y.; He, Z.; Luo, F.; Zhou, J. Molecular ecological network analyses. BMC Bioinformatics 2012, 13, 113, DOI:10.1186/14712105-13-113.

47. Du, Z.J.; Wang, Z.J.; Zhao, J.X.; Chen, G.J. Woeseia oceani gen. nov., sp nov., a chemoheterotrophic member of the order Chromatiales, and proposal of Woeseiaceae fam. nov. Int $J$ Syst Evol Micr 2016, 66, 107-112, DOI:10.1099/ijsem.0.000683.

48. Mussmann, M.; Pjevac, P.; Kruger, K.; Dyksma, S. Genomic repertoire of the Woeseiaceae/JTB255, cosmopolitan and abundant core members of microbial communities in marine sediments. Isme $J$ 2017, 11, 1276-1281, DOI:10.1038/ismej.2016.185. 
49. Martiny, J.B.; Bohannan, B.J.; Brown, J.H.; Colwell, R.K.; Fuhrman, J.A.; Green, J.L.; Horner-Devine, M.C.; Kane, M.; Krumins, J.A.; Kuske, C.R.; et al. Microbial biogeography: putting microorganisms on the map. Nat Rev Microbiol 2006, 4, 102-112, DOI:10.1038/nrmicro1341.

50. Gonnella, G.; Bohnke, S.; Indenbirken, D.; Garbe-Schonberg, D.; Seifert, R.; Mertens, C.; Kurtz, S.; Perner, M. Endemic hydrothermal vent species identified in the open ocean seed bank. Nat Microbiol 2016, 1, 16086, DOI:10.1038/nmicrobiol.2016.86.

51. Fierer, N.; Jackson, R.B. The diversity and biogeography of soil bacterial communities. Proc Natl Acad Sci U S A 2006, 103, 626-631, DOI:10.1073/pnas.0507535103.

52. Tazi, L.; Breakwell, D.P.; Harker, A.R.; Crandall, K.A. Life in extreme environments: microbial diversity in Great Salt Lake, Utah. Extremophiles 2014, 18, 525-535, DOI:10.1007/s00792-014-0637-x.

53. Ley, R.E.; Harris, J.K.; Wilcox, J.; Spear, J.R.; Miller, S.R.; Bebout, B.M.; Maresca, J.A.; Bryant, D.A.; Sogin, M.L.; Pace, N.R. Unexpected diversity and complexity of the Guerrero Negro hypersaline microbial mat. Appl Environ Microbiol 2006, 72, 3685-3695, DOI:10.1128/AEM.72.5.3685-3695.2006.

54. Sorensen, K.B.; Canfield, D.E.; Teske, A.P.; Oren, A. Community composition of a hypersaline endoevaporitic microbial mat. Appl Environ Microbiol 2005, 71, 7352-7365, DOI:10.1128/AEM.71.11.7352-7365.2005.

55. Tsiamis, G.; Katsaveli, K.; Ntougias, S.; Kyrpides, N.; Andersen, G.; Piceno, Y.; Bourtzis, K. Prokaryotic community profiles at different operational stages of a Greek solar saltern. Res Microbiol 2008, 159, 609-627, DOI:10.1016/j.resmic.2008.09.007.

56. Benlloch, S.; Lopez-Lopez, A.; Casamayor, E.O.; Ovreas, L.; Goddard, V.; Daae, F.L.; Smerdon, G.; Massana, R.; Joint, I.; Thingstad, F.; et al. Prokaryotic genetic diversity throughout the salinity gradient of a coastal solar saltern. Environ Microbiol 2002, 4, 349-360.

57. Lozupone, C.A.; Knight, R. Global patterns in bacterial diversity. Proc Natl Acad Sci U S A 2007, 104, 11436-11440, DOI:10.1073/pnas.0611525104.

58. Liu, J.; Fu, B.; Yang, H.; Zhao, M.; He, B.; Zhang, X.H. Phylogenetic shifts of bacterioplankton community composition along the Pearl Estuary: the potential impact of hypoxia and nutrients. Front Microbiol 2015, 6, 64, DOI:10.3389/fmicb.2015.00064.

59. Blodau, C. A review of acidity generation and consumption in acidic coal mine lakes and their watersheds. Sci Total Environ 2006, 369, 307-332, DOI:10.1016/j.scitotenv.2006.05.004. 


\section{Figure Legends}

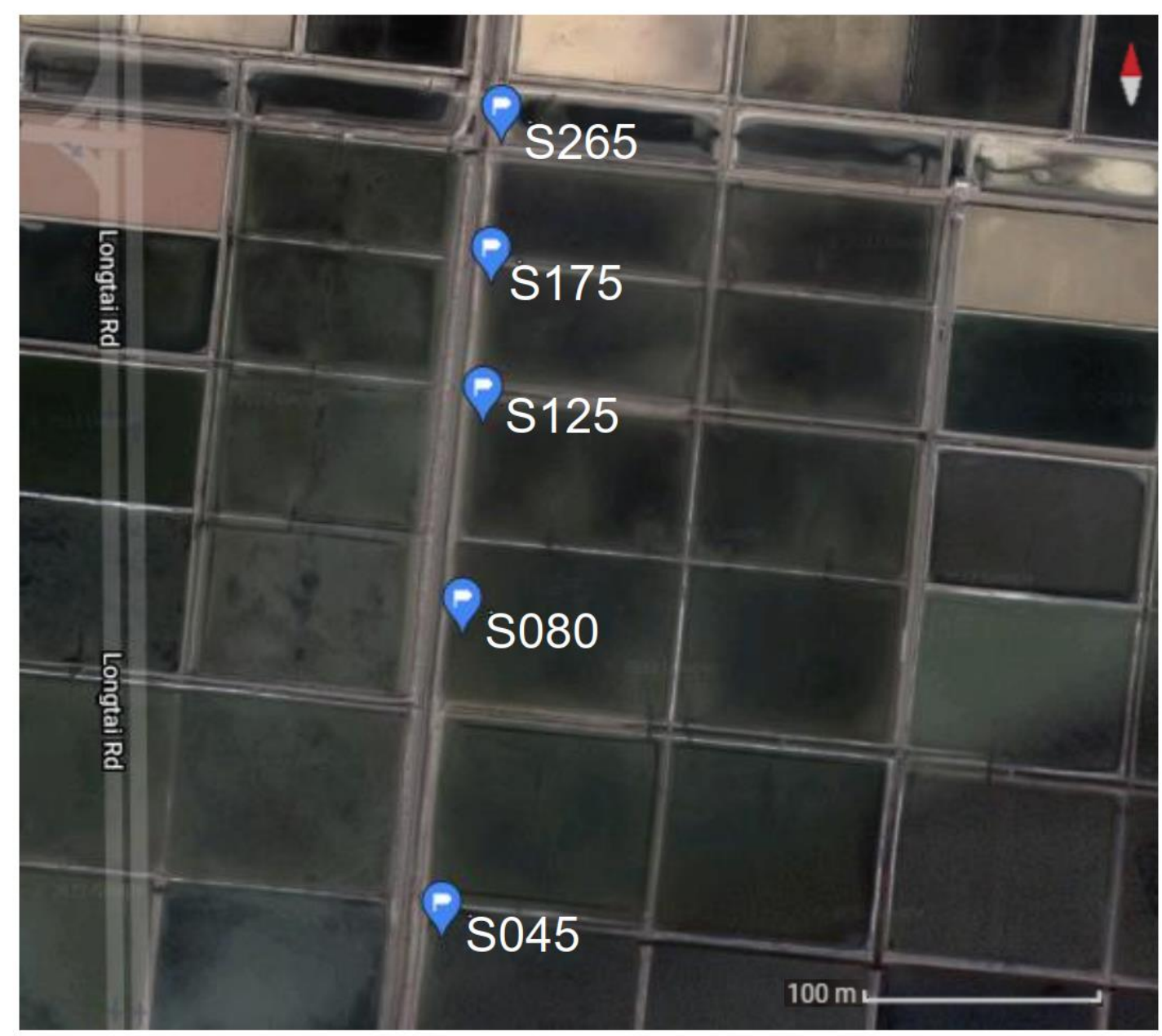

Figure 1. Overview of the Wendeng salterns.

The salterns were made up by a set of shallow ponds. The salinity of these shallow ponds was about 40, 80, 125, 175 and $265 \mathrm{~g} / \mathrm{L}$ in sampling sites S045, S080, S125, S175 and S265, respectively. 


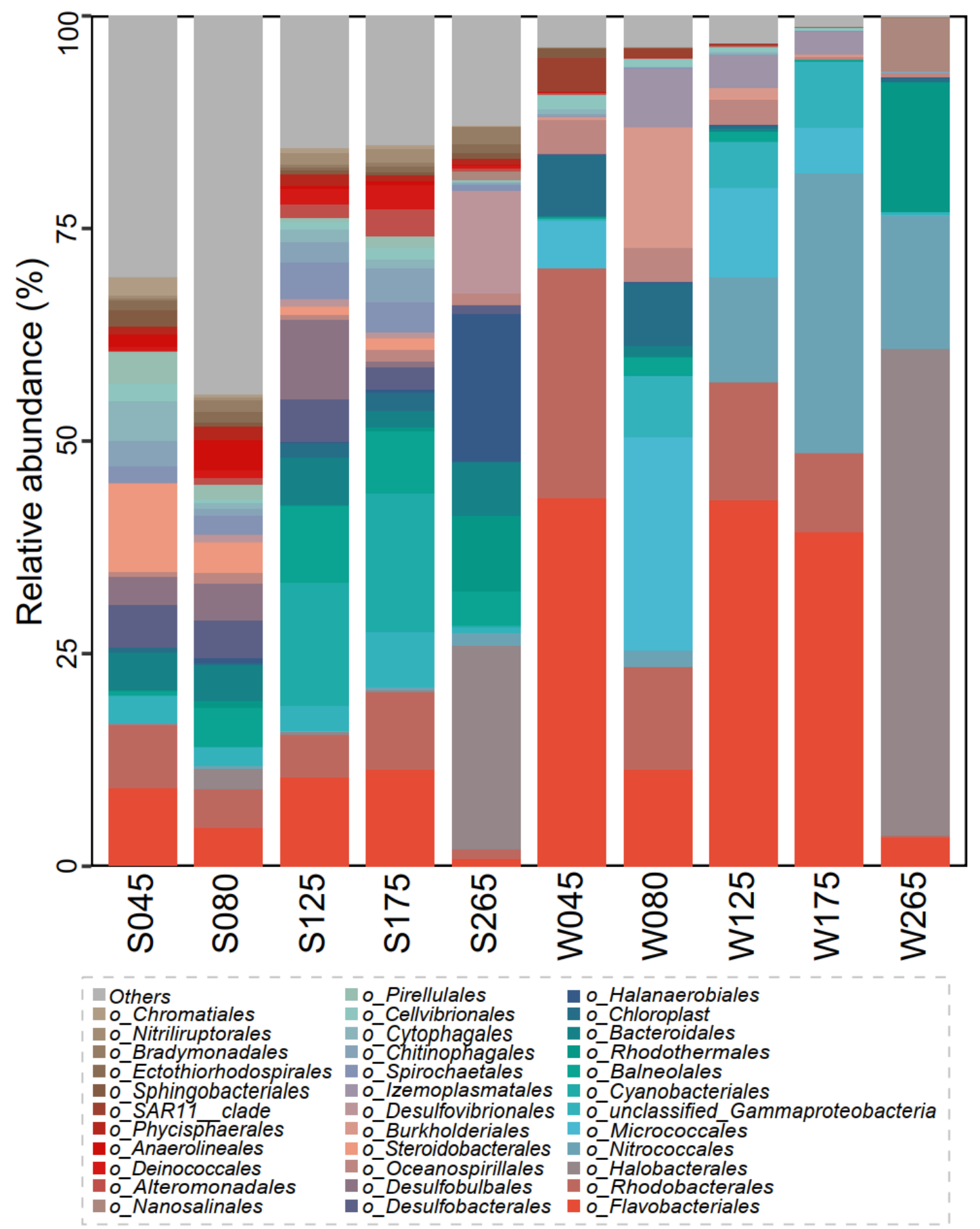

Figure 2. Prokaryotic community composition as the abundant order along a gradient of increasing salinity.

The top 35 abundant order taxa were shown. The labels S and W represented sediment and water samples, respectively. The number of each sample means salinity. 

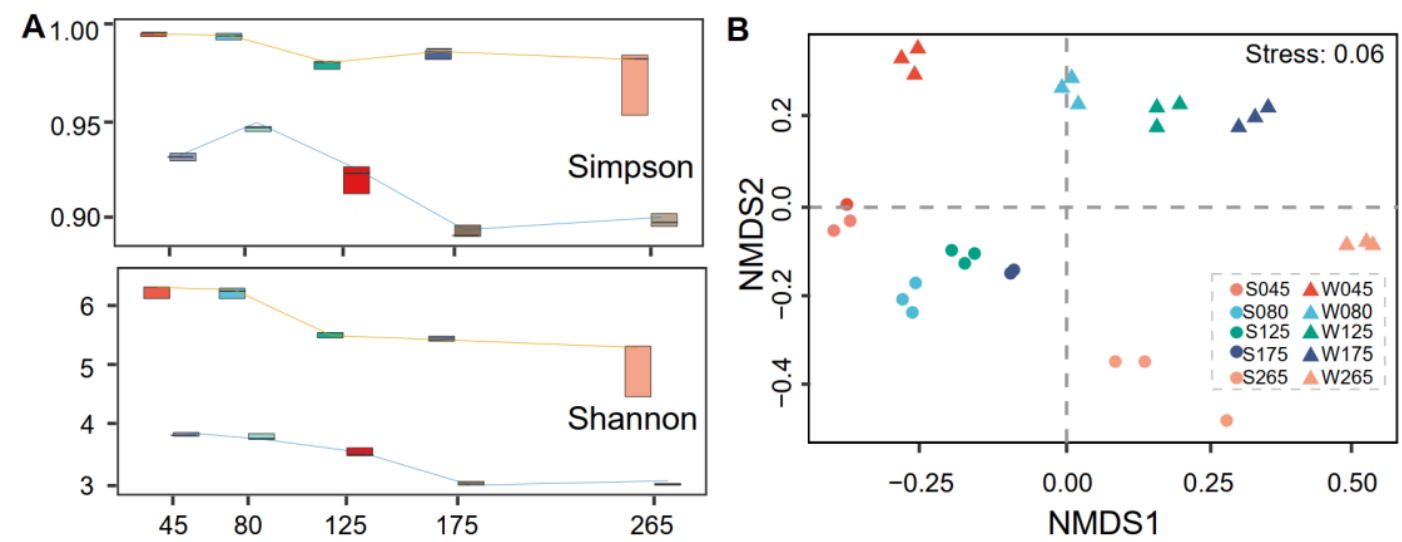

Figure 3. The biodiversity of prokaryotic community.

A, The alpha diversities (simpson and Shannon index) were analyzed by R package (microeco) and displayed along a gradient of increasing salinity. The orange and blue lines represented sediment and water groups in salterns, respectively. B, Non-metric multidimensional scaling (NMDS) were analyzed to exhibited beta-diversity of prokaryotic community in salterns. 
A
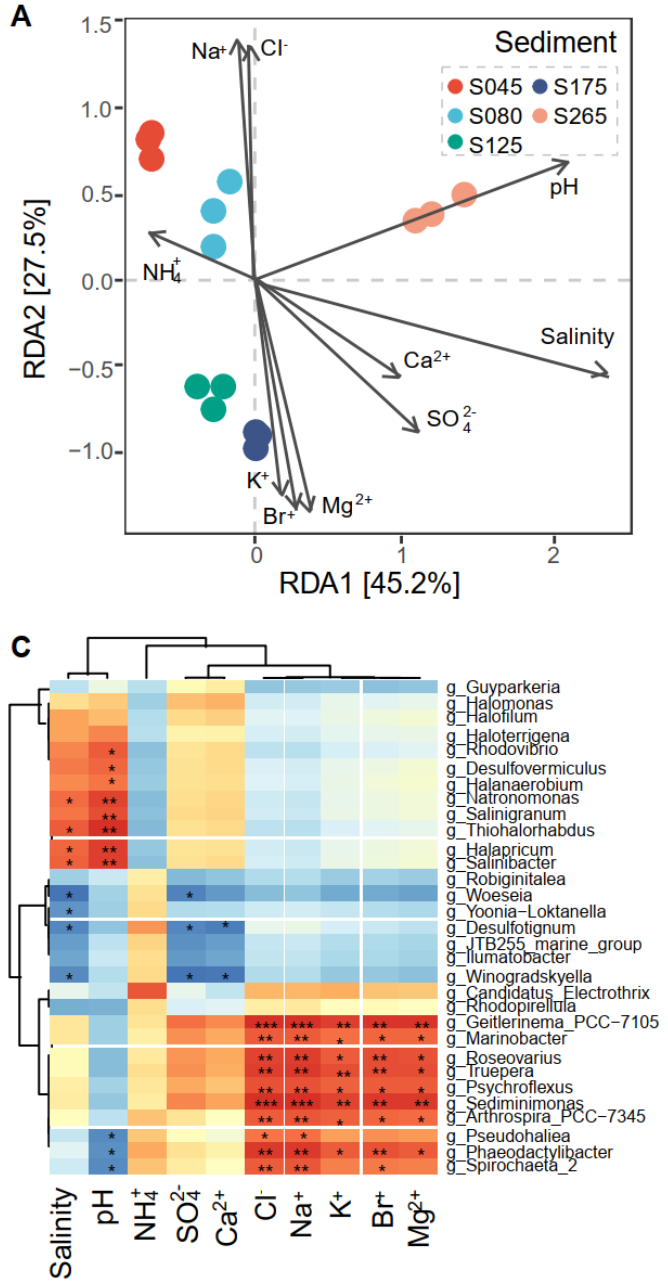

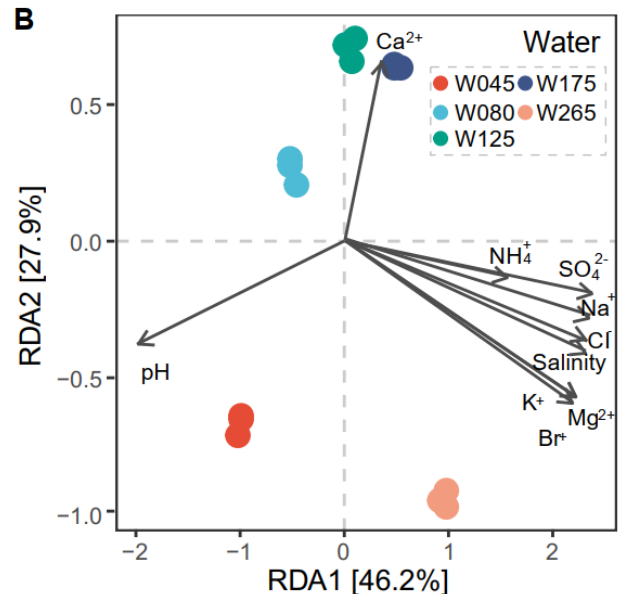

D

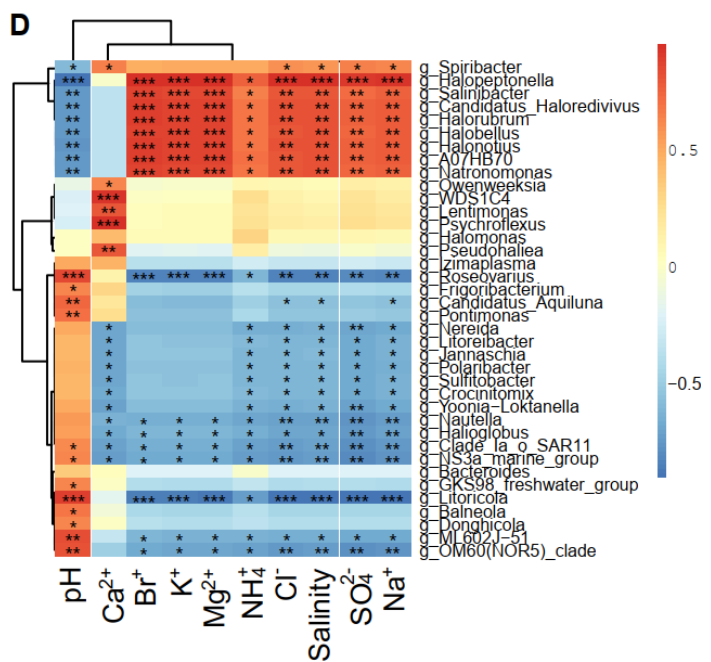

Figure 4. The relationships between physicochemical factors and prokaryotic community composition.

A-B, Redundancy analysis (RDA) between prokaryotic community bray-distance and physicochemical factors were employed both in sediment and water samples of salterns.

C-D, The strong-related genus taxa with physicochemical factors were shown (C, sediment groups; D, water groups). Significant differences were marked. $\left({ }^{*} 0.01<P \leq\right.$ $\left.0.05,{ }^{* *} 0.001<P \leq 0.01,{ }^{* * *} P \leq 0.001\right)$. 
B

Water

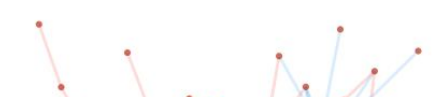

A

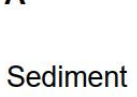

$\therefore$

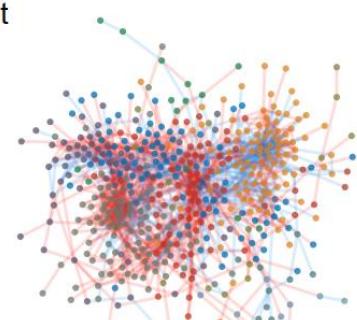

$\because \because \therefore$
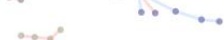
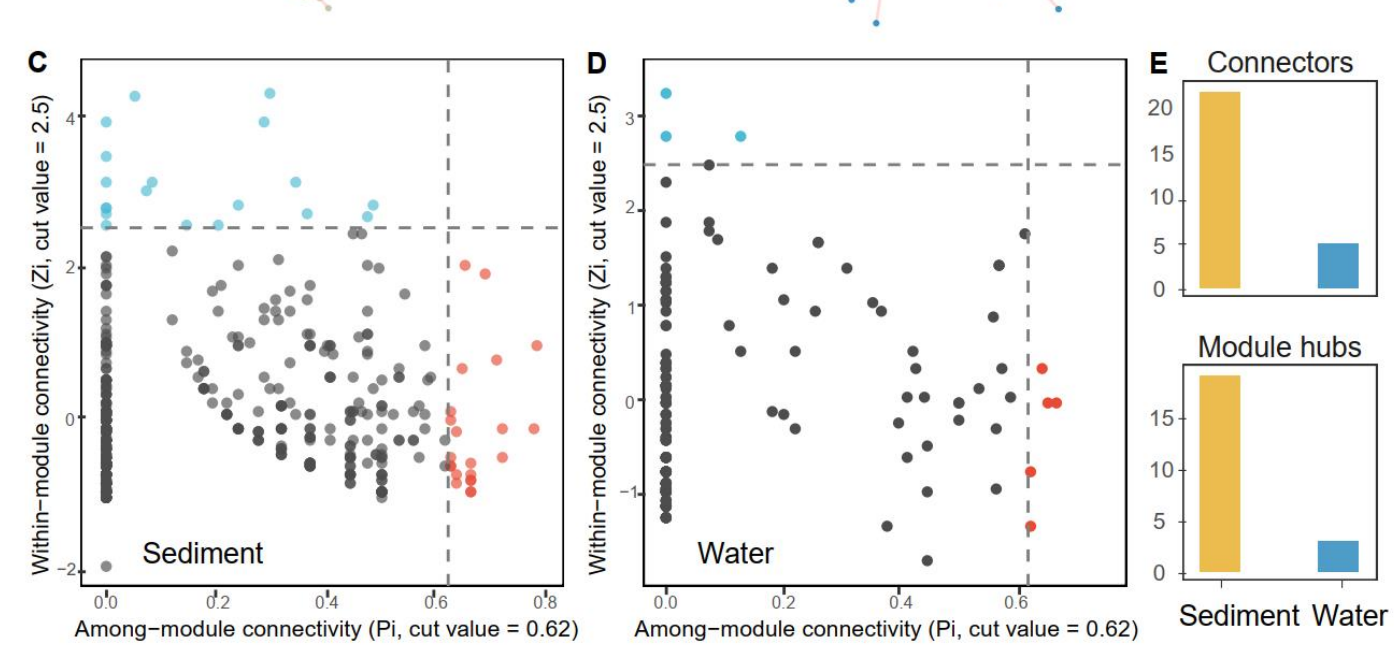

Figure 5. Salterns networks between sediment and water groups.

A-B, Visualization of the microbial MENs in sediment and water groups. Modules were randomly colored. The red and blue links between nodes represented positive and negative relationships, respectively. C-D, The role of OTUs in network communities were determined by within-module connectivity ( $\mathrm{Zi}$ ) and among-module connectivity (Pi). The light blue and red points represented module hubs and connectors, respectively. E, Summarization of keystone taxa (including module hubs and connectors). 


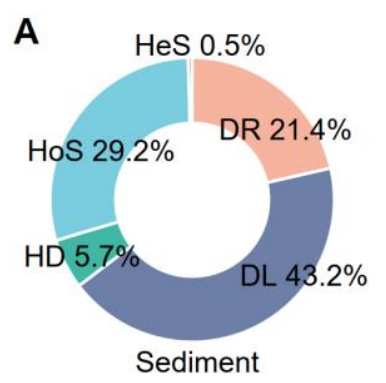

B

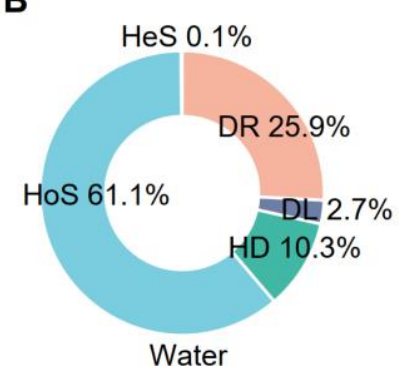

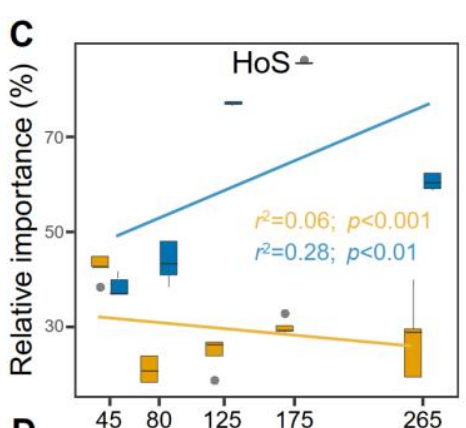
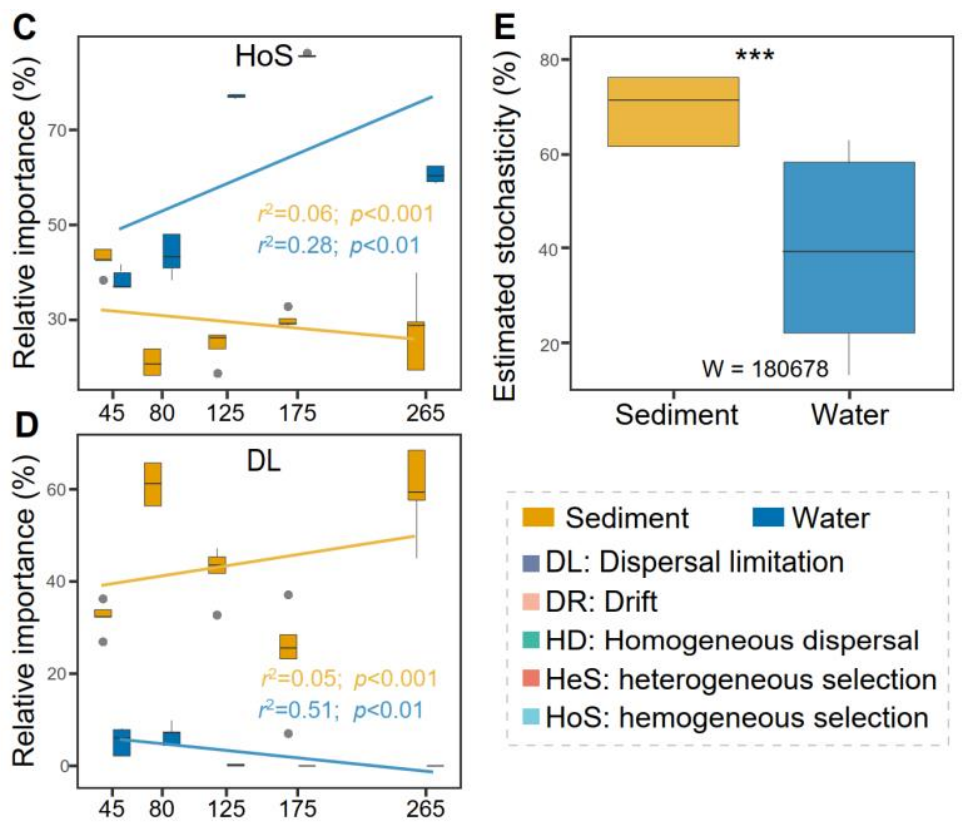

Sediment Water

DL: Dispersal limitation

DR: Drift

nD: Homogeneous dispersal

- HeS: heterogeneous selection

- HoS: hemogeneous selection

Figure 6. Relative importance of different ecological processes in salterns sediment

and water.

A, Relative importance of different ecological processes in sediment samples. B, Relative importance of different ecological processes in water samples. C-D Changes of homogeneous selection and dispersal limitation in sediment (orange box) and water (blue box). The adjusted $r^{2}$ and $P$ values from linear regressions are shown. $\mathbf{E}$ Stochasticity estimated both in sediment and water samples. Mann-Whitney U test results were shown and significance was expressed as ${ }^{* *} P \leq 0.001$. 